\title{
2D QUALITATIVE SHAPE MATCHING APPLIED TO CERAMIC MOSAIC ASSEMBLY
}

\author{
Lledó Museros $^{(1)}$, Zoe Falomir ${ }^{(1)}$, Francisco Velasco $^{(2)}$, Luis González-Abril ${ }^{(2)}$, Isabel Martí ${ }^{(1)}$ \\ ${ }^{(1)}$ Department of Computer Sciences and Engineering. Universitat Jaume I Castellón. Avda. \\ Vicent Sos Baynat s/n E-12071 Castellón (Spain) \\ Phone: +34964728283 Fax: +34964728486
}

museros@icc.uji.es ; zfalomir@icc.uji.es; Isa.Marti@uji.es

${ }^{(2)}$ Department of Applied Economics I. University of Seville. Av. Ramón y Cajal $n^{\circ} 1 . E-41018$ Sevilla (Spain)

Phone: +34954554345 Fax: +34954551636

luisgon@us.es; velasco@us.es

\begin{abstract}
A theory of shape recognition of 2D objects and its application in the ceramic industry for intelligent automation of the mosaic mural assembly process are presented in this paper. This theory qualitatively describes the shapes of the objects, considering: i) shape boundary characteristics, such as angles, relative length, concavities, and curvature; and ii) their color and size. The shapes to be recognized may be regular or irregular closed polygons, or closed curvilinear figures. Each figure is described as a symbolic character string that contains all its distinctive characteristics. This description is used to determine whether the shape of two figures matches. Then, given a design of a mosaic and given a set of physical ceramic tesserae, an application is developed in order to recognize the tesserae that form the mosaic, thus enabling the intelligent and automated assembly of ceramic mosaics.
\end{abstract}

Keywords: Qualitative reasoning, Cognitive Vision, Industrial Robotics.

\section{Introduction}

A mosaic is a decorative art form in which small ceramic tiles (tesserae) are assembled to form a predefined image. There are two main methods of creating mosaics, called "direct method" and "indirect method" (Dierks 2004). The direct method consists of directly adhering the individual tesserae on the substrate surface, and it is more appropriate when the surfaces to be covered with the mosaic have a three-dimensional shape. In addition, it has the further advantage of progressively showing how the mosaic is materializing as it is being created, so that the necessary fixing and color adjustments in the tesserae can be made immediately. But it also has some disadvantages: i) the artist needs to work on the surface to be covered, which is often impracticable during long periods of time (e.g. owing to bad weather); ii) it is inappropriate in large-scale projects; and iii) it is difficult to control finished surface uniformity, which is very important when creating a functional surface such as flooring.

In these situations, the indirect method is used since it consists of previously assembling and pre-arranging the tesserae on various grids that are subsequently fixed onto the surface to be clad with the mosaic. The grids are installed according to a specific plan, in order to create a particular decorative image. Each grid also follows a plan or design for arranging the individual tesserae, so that when the grids are joined they will create the desired image.

Both methods produce hand-made mosaics, so they have a high manufacturing cost. Hence, with the aim of reducing costs, an application has been designed to enable the development of a complete system for the intelligent automation of constructing ceramic mosaics by the 
indirect method. To achieve this objective, a qualitative approach for 2D shape matching was the most appropriate, because it allows uncertainty to be dealt with efficiently, for example, the uncertainty associated with the fact that there are no two exactly identical tesserae, or the uncertainty associated with working under industrial conditions.

The rest of this paper is organized as follows: a brief overview of the main qualitative theories of shape recognition is presented in Section 2. The qualitative theory of shape matching implemented for recognition of the tesserae is given in Section 3. The image processing software developed is described in Section 4. The laboratory prototype developed is shown in Section 5, and the conclusions and future work are presented in Section 6.

\section{State of the art in qualitative theories of shape recognition}

Shape perception and identification is a task carried out by human beings in many of their activities. We relate shapes to objects and define them as the description of the boundary of those objects. However, cognitively speaking, a shape is considered as an aspect of a stimulus that remains invariant despite changes in size, position and orientation (Goldstone, 1999).

Although shape is one of the most important features of an object, it is particularly difficult to describe computationally since, when a three-dimensional object is projected from the real world onto a two-dimensional image plane, one dimension of the object information is lost. Therefore, the shape extracted from a digital image of the object partially represents the projected object. Moreover, digital images are often corrupted with noise, defects, arbitrary distortion and occlusion. Furthermore, shape identification or shape matching is also a difficult task to achieve if this identification has to be independent of size, position and orientation, just as it is in human vision.

Most of the image processing in computer vision has been carried out by applying quantitative techniques to describe and identify the shape of the objects contained in an image. In (Mehtre, Kankanhalli and Lee, 1997), these techniques are divided into two categories: boundary-based and region-based methods. Boundary-based methods describe shapes by only using the contour or border of the object, while region-based techniques take into account details of the interior of the object. Boundary-based methods (Rucklidge, 1997; Belongie, Malik, and Puzicha, 2001; Zhang and Lu, 2002, Giannarou and Stathaki 2007) are more popular than region-based methods (Lu and Sajjanhar, 1999; Morse, 1994) because human beings are thought to discriminate shapes mainly by their contour features. Another reason is that in many of the applications, the shape contour is the only feature of interest, while the interior content is not important. However, in applications where internal details are as important as the contour, the use of a contour-based approach is not sufficient.

The problem of shape description and identification is tackled qualitatively in this paper since it is thought that this uses a process closer to that used by human beings to describe images. So meaning can be added to the qualitative concepts used for image description and then this can be used for reasoning. The theory proposed can be considered as both region- and boundary-based since it describes the boundary of objects and their interior content, such as holes and color. Qualitative representation schemes are most useful for applications of Artificial Intelligence concerned with the identification and classification of objects that involve reasoning of shapes. Qualitative shape descriptions focus on relevant and invariant features of shape that are considered most important within a particular context (Galton and Meathrel 1999). Viewed more broadly, the origin of qualitative descriptions is probably that some processes are carried out to derive a high-level description based on low-level inputs. 
Looking at existing approaches to qualitative shape representation, there are two main categories, volume- or region-based approaches and outline- or boundary-based approaches, as in the case of quantitative ones. In the first category, a volume-based approach, 3D-model representation using simple cylindrical primitives was developed by Marr and Nishihara (1978). Another volume-based approach, known as geon-based representation, was developed by Biederman (1987). Biederman stated that a complex solid object can be described with a number of smaller solid pieces put together in a particular manner. In contrast, the second category, boundary-based approaches, rely more on the 2D domain. These approaches characterize the form of the outline of a shape, considering the variation in the curvature of its boundary across different positions. In this category there are several approaches (Hoffman and Richards 1985; Leyton 1988; Leyton 2005; Jungert 1993) and more recently the qualitative outline theory by Galton and Meathrel (1999) and tripartite line tracks by Gottfried (2005) are significant.

Some boundary-based approaches for the description and matching of 2D shapes (Bennett 1994; Cohn 1995; Clementini and Di Felice 1997; Chase 1997) rely on topology and/or logics. For instance, Bennett's work uses the concept of convex-hull of a region, that is, the smallest convex region of which the shape of the object is a part. Then, shapes are classified as similar if their convex hulls are similar. Cohn (1995) developed a region-based approach to describe shape, inspired by a logical theory of space called the Region Connection Calculus. A shape is then described by the number of concavities that the shape has and with the regional connection relations that hold between each pair of concavities. A set of relation predicates defined by Cohn is used to distinguish between different shapes where the same relations hold between concavities and they are adjacent.

Hoffman and Richards (1982 and 1985) introduced the contour codons technique for describing smooth planar curves for recognition purposes. A contour codon is a piece of a curve that is circumscribed by curvature minima and that can contain either zero, one or two points of zero curvature. Five codon types are defined, reflecting how many zero curvature points are present in a segment of curve. This approach can only represent a limited scope of shapes, all of them containing open and closed curves. Thus, Rosin (1993) further extended this approach in the context of multi-scale representation and matching of curves. He supplemented the original five codons with a large number of codons to support the description of curves that contains straight-line segments and tangent discontinuities. Nevertheless, this approach still cannot distinguish between circles and ellipses.

A different approach was introduced by Leyton (1988), where curves can be divided into smaller curves based on their symmetry axis and can be described in terms of curvature extrema. Another qualitative boundary-based shape representation scheme was developed by Galton and Meathrel (2000). They describe the outline of a shape qualitatively by seven curvature-type alphabets. Consequently a shape can be qualitatively represented by a string of these curvature-type alphabets under some specified grammar rules. Galton and Meathrel impose some constraints in their scheme, for example, a shape must not contain two consecutive occurrences of the same curvature-type symbol. Again, this limits the scope of the representation scheme.

There is also a set of approaches that may be described as point-based representation, because they represent different aspects of the shape by describing reference points. Within this group there is the approach developed by Jungert (1993), which describes the shape of an object as symbolic projection strings that are generated from projections of the objects down to the coordinate axis. Another approach inside this group is based on tripartite line tracks (TLT), a set of qualitatively distinct polygons (Gottfried 2005). This approach uses a reference point 
orientation grid (Freksa and Zimmermann 1992) to create a set of 36 different tripartite line polygons, which is reduced to 12 by removing all symmetrical cases. This scheme has two limitations: i) it cannot represent curves and circular shapes; and ii) each TLT arrangement can vary significantly at its end points within the same TLT concept, which means that there are shapes that the scheme cannot distinguish between.

Analyzing the approaches presented shows that in the group of topology-based approaches eleven convex shapes have the same description, and moreover it is not straightforward to describe polygons. Hoffman and Richard's contour codon, and Leyton's initial set of extreme primitives are limited to describing smooth, continuously differentiable, closed curves. Galton and Meathrel's representation cannot distinguish some cognitively distinguishable shapes. The approach by Gottfried is not able to describe smooth curvilinear shapes and it also has the problem of being unable to distinguish cognitively distinguishable shapes.

Therefore, for the mosaic assembly application and to reduce as much as possible some of the drawbacks of the approaches presented, especially the problem of not distinguishing between cognitively distinguishable shapes, a 2D qualitative description and matching theory has been developed (Museros and Escrig 2004). This approach may be considered a reference pointbased representation, since the theory uses information on the vertices and maximum curvature points to describe an object's shape. It is worth noting that the theory developed also takes into account the relative length of the edges as well as the size and color of the objects as relevant characteristics in the recognition process. These aspects had not been considered in previous studies.

\section{The 2D qualitative theory of shape recognition}

The architecture of the 2D qualitative approach for shape description (2DQSR) and identification is described in Figure 1. The inputs to the 2DQSR can be: (i) information about objects contained in a digital image; or (ii) information about objects obtained from a design. From either of these inputs, the 2DQSR obtains the relevant points of the boundaries of the objects, which are the vertices and the point of maximum curvature of each curve. Once the relevant points are detected, the 2DQSR uses them to give the qualitative description of the shape. This theory considers qualitatively the angles, relative edge length, concavities and convexities, and types of curvatures of the boundaries of the objects, and it also considers qualitatively, the color and size of the objects. The shapes recognized are regular and irregular closed polygons that can have curve segments or curvilinear shapes, and these shapes can contain holes. To describe shapes with holes, topological and qualitative spatial orientation aspects are considered in order to relate the hole to its parent object. When all the holes are described and related to their parent objects, general information about the shape of each object is added, such as the color of the object and other qualitative labels indicating if the shape contains curves/holes or not. As the result of the description process, each object is represented by a string describing its qualitative distinguishing features. Finally, a matching process was defined that determines if two objects have the same size, shape and color or not by comparing the symbolic descriptions obtained. An important contribution of this paper is to unite all the qualitative features of boundary description given above in a unique symbolic description. In the rest of this section the architecture is explained with more details. 


\section{Description of regular and irregular polygonal objects}

The qualitative description of regular and irregular polygonal objects is based on the qualitative description of each reference point, named $i$, which is determined using the previous reference point, named $i-1$, and the following reference point, named $i+1$ in a clockwise direction.

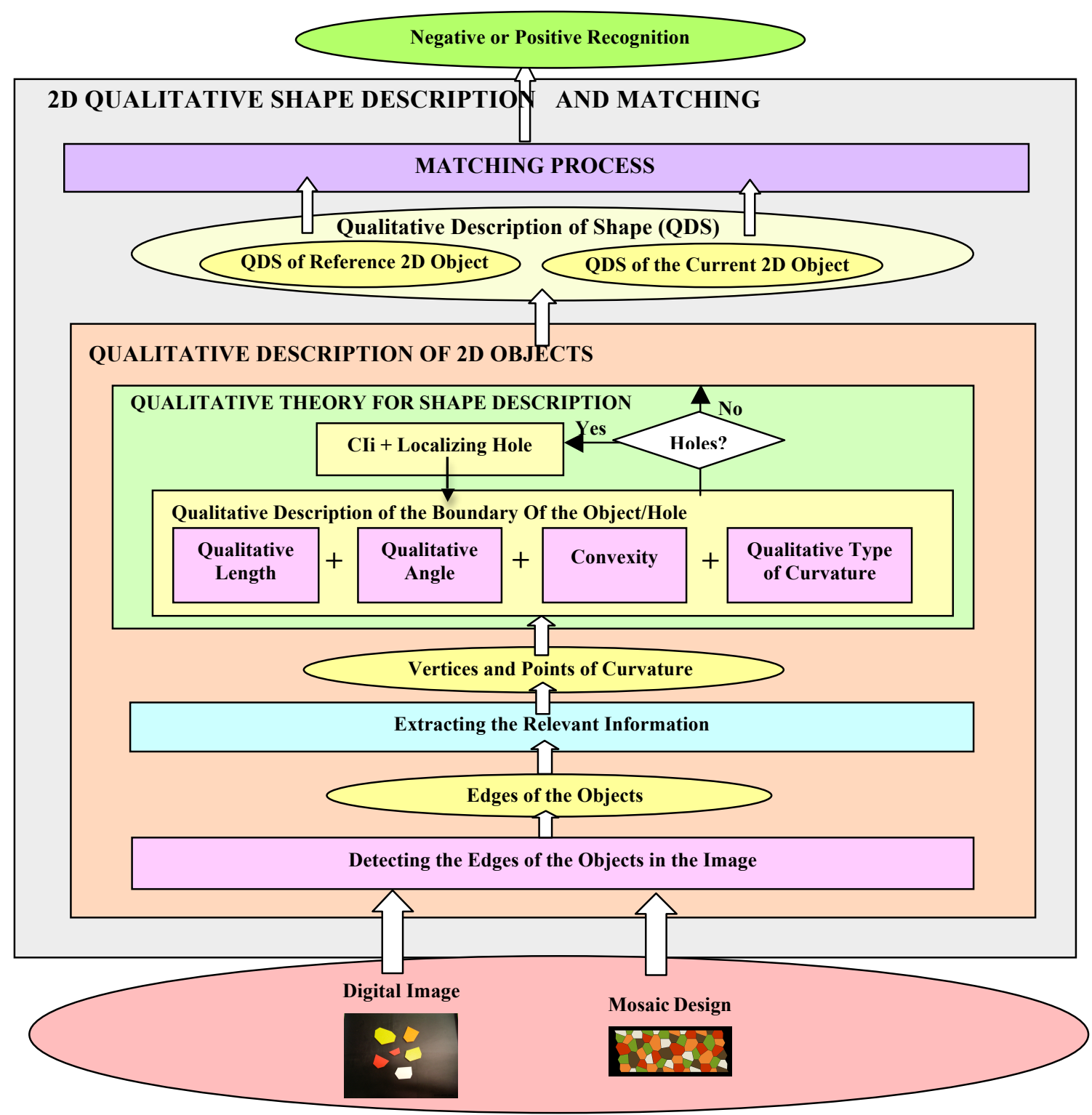

Fig. 1 Architecture of the 2D qualitative shape-matching method applied to ceramic mosaic assembly

The description of each reference point is given by a set of three elements (triple) which is $<A i, C i, L i>$, where $A i$ represents the angle at the reference point $i, C i$ represents the type of convexity of point $i$ and $L i$ represents the relative length of the edges associated with reference point $i$ (edge formed by vertices $i-1$ and $i$ versus edge formed by vertices $i$ and $i+1$ ). For the sake of clarity, the following labels have been chosen: $\mathrm{Ai} \in\{$ right-angled, acute, obtuse $\} ; \mathrm{Ci}$ $\in\{$ convex, concave $\}$; and $L i$ is a member of LRS, where LRS $=\{$ smaller, equal, bigger $\}$. 
Convexity: The convexity of the point $i$ is determined as follows: if $V i$ means vertex $i$, and $V i$ wrt $V i-1 V i+1$ means the relation of $\mathrm{V} i$ with respect to (wrt) the oriented line from $V i-1$ to $V i+1$, then: If Vi wrt $V i-1 V i+1 \in$ left (right) then Vi is convex (concave).

Angle:The description of the angle of the vertex $V i$ is determined as follows: if $C_{i-1 i+l}$ denotes the circle with a diameter of $V i-1 V i+1, \delta C_{i-1 i+1}$ denotes its boundary and $C_{i-1 i+1}{ }^{o}$ denotes its interior $\left(C_{i-1 i+1}-\delta C_{i-1 i+1}\right)$ then the angle of $V i$ is calculated: If $V i \cap \delta C_{i-1 i+1} \neq \varnothing$ then Vi is rightangled, else if Vi $\cap C_{i-1+1}{ }^{o} \neq \varnothing$ then Vi is obtuse, otherwise Vi is acute.

The relative length: a length model has been developed, based on the models given in Escrig and Toledo (1998), which compares the lengths of two consecutive edges as follows: if the Euclidean distance is considered, then the compared length assigned to the vertex I is: If $d(V i$ $1, V i)<d(V i, V i+1)$ then smaller, else if $d(V i-1, V i)>d(V i, V i+1)$ then bigger, otherwise equal.

\section{Description of objects with curves}

In this case, the description of each reference point is given by a triple $\langle$ Curve, $C i, T C i>$, where the symbol Curve means that the node in the description string is describing a curve, $\mathrm{C} i$ means the type of convexity of point $i$ and $T C i$ means the type of curvature of the curve associated with the point $i$, where the following labels have been chosen: $C i \in\{$ convex,concave $\}$, and $T C i \in\{$ plane,semicircle,acute $\}$.

Therefore, first of all the symbol curve is set to indicate that the next node in the qualitative description corresponds to the description of a curve. To qualitatively describe a curve, 3 points are used: the initial and final points of the curve and the point of maximum curvature of the curve; these are obviously consecutive points. The description, however, is associated only with the point of maximum curvature, and the other two points are used only to calculate the description of the curve (see Figure 2 where point $\mathrm{j}$ is characterised). Thus, the convexity of the point $j$ is determined using the oriented line from $i$ to $k$, as follows: If $V j$ wrt $V i V k \in l e f t$ (right) then $\mathrm{Vj}$ is convex (concave).

The type of curvature (TCj) of the point $\mathrm{j}$ (point of maximum curvature) is determined by first calculating the point Pik, which is the halfway-point of the line between $i$ (initial point of the curve) and $k$ (final point of the curve). Next, the distance between vertex $i$ and Pik, named $d a$, and the distance between vertex $j$ and $P i k$, named $d b$, are calculated. By comparing both distances $T C j$ is determined as follows (see Figure 2):

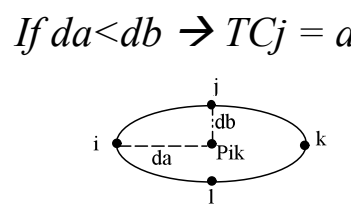

a)

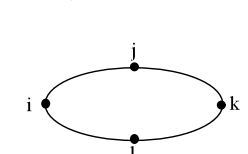

b)

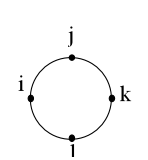

c)

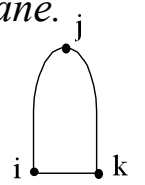

d)

Fig. 2 a) Distances calculated for determining $T C j$, b) $T C j=$ plane, c) $T C j=$ semicircle, d) $T C j=$ acute

\section{Description of objects with holes}

The Completely Inside Inverse (CIi) topological concept is used in order to describe objects with holes, because a hole is always inside the boundaries of a closed object. The relation $C I i$ is defined as the inverse of the $C I$ (Completely Inside) relation, which is defined in turn from the formal definition of the in relation as follows: $(h 1, i n, h 2) \leftrightarrow h 1 \cap h 2=h 1 \wedge h^{\circ} 1 \cap h^{\circ} 2 \neq \varnothing$ 
If $(h 1, i n, h 2)$ is obeyed, the following algorithm distinguishes between three possible relations: equal, touching-from-inside, and completely-inside: If (h2,in,h1) then (h1,equal,h2). But if h1 $\cap \delta h 2 \neq \varnothing$ then (h1,touching-from-inside, h2). Otherwise (h1,completely-inside, h2).

Therefore, for describing an object with holes the following steps are taken:

1. The qualitative shape description of the exterior boundary of the object (parent object) is constructed following the steps described in previous sections.

2. Then the qualitative shape description of the boundary of each hole is constructed.

3. Each hole and the parent object are related by adding two types of information: i) The topological relation between the parent object and each hole is set. In the case of closed objects the parent object is always Completely Inside Inverse (CIi) of the holes; and ii) The position of each hole inside the parent object is set using Frank's Cardinal Reference System (1992) (CRF), which divides space into eight cones (see Figure 3). The CRF is defined by placing its origin at the centroid calculated with the definition of the centroid of a closed regular or irregular polygon given in (Steger 1996). In the case of curvilinear shapes or shapes that contain curve segments, these shapes are approximated to polygonal shapes in which the vertices are the reference points considered for the qualitative description of the shape and these vertices are joined by rectilinear segments.

The position that occurs when the hole is placed around the centroid, and all positions hold is called centre (C). When several positions hold for a given hole, then the position is set to a set of all these positions (see Figure 3). Then once the CRF is placed in the object, the position of the hole with respect to the object is calculated. For instance, the position of the hole with respect to the parent object in Figure $3 \mathrm{a}$ is $[\mathrm{SE}, \mathrm{S}]$.

\section{Complete description of an object}

The complete description of a 2D object is defined by the following tuple:

[type_hole, type_curve, [Color, [A1, C1, L1 | Curve, C1, TC1] ... [An, Cn, Ln |Curve, Cn, TCn]], CIi, Position, [type_curve, [AH1, CH1, LH1 |Curve, CH1, TCH1] ... [AHj, CHj, LHj | Curve, $\mathrm{CHj}, \mathrm{TCHj}] \mathrm{J}$, where $n$ is the number of reference points of the parent object and $j$ is the number of reference points of the hole. type hole symbol may have one of the following values [without holes, with holes], and the symbol type_curve may have one of the following values [without curves, with curves, only curves]; both symbols are introduced to accelerate the correspondence or recognition process. Color is the RGB color of the figure described by the three basic components, the Red, Green, and Blue coordinates.

Each vector, [Ai, $\mathrm{Ci}, \mathrm{Li} \mid \mathrm{Curve}, \mathrm{Ci}, \mathrm{TCi}]$, represents a qualitative description node. $\mathrm{CI} i$ is the topological relation that relates the hole to its parent object. Finally, Position is one or a set of orientation relations given by the CRF, in order to provide the relative position of the hole with respect to its parent object. CIi, the Position, and the description of the hole boundaries are only used when the object is of the "with holes" type and these will appear as many times as there are holes in the figure.

It is important to notice that the description of the boundary of a hole is always done by taking as first vertex to describe the one closest to the initial vertex of the parent object description. This is because it is subsequently needed in the correspondence process in order to perform a non-cyclical comparison of the hole boundaries, where it is necessary to ensure that the description always begins from the same vertex. The final vector with the characteristics of the figure is thus constructed. 


\section{The matching process}

The matching process is carried out as follows: first one of the objects is taken as reference and the other is considered the shape to be compared and matched. Then, qualitative areas of both objects are compared, and if they match, the description of the object taken as reference is constructed. Next, the qualitative description of the other object is constructed but only up to the description of the parent object. These two strings are compared if both strings are of the same type (with or without holes), same color (in a qualitative way) and the parent objects are equal. If all these features match then the position and boundary description of the holes are compared. If all the holes have the same position and boundary, the objects are considered equal.

With respect to the area: This is compared in a qualitative way. The threshold for deciding that two shapes are the same is given by the application itself, since in the context of a mosaic, it is determined by the joint between tiles (space left between two tiles when they are assembled). As the joint differs from one type of design to another, this value is set by the user of the application. Then if the difference between the areas of the tiles is less than the joint size, the shapes can represent the same object.

The colors were considered to be always solid colors, and it is calculated the Delta E distance between two colors $C 1=(R 1, G 1, B 1)$, and $C 2=(R 2, G 2, B 2)$ in RGB, which is the Euclidean distance. If the Delta_E is less than 0.2 then colors are considered the same because a human eye experienced in recognizing the color field cannot differentiate between these two colors.

To compare the parent objects, the algorithm ComparingVertices (algorithm 1) is applied. It is a cyclical-order matching algorithm and given two sets of vertices, it returns if both strings are equal cyclically or not, and the vertex in the second object that corresponds to vertex number 1 , which is the uppermost (left) vertex of the first shape. If both sets are not equal, the vertex in the second set is not found (a value of -1 is assigned). If the objects do not have holes, the process finishes here.

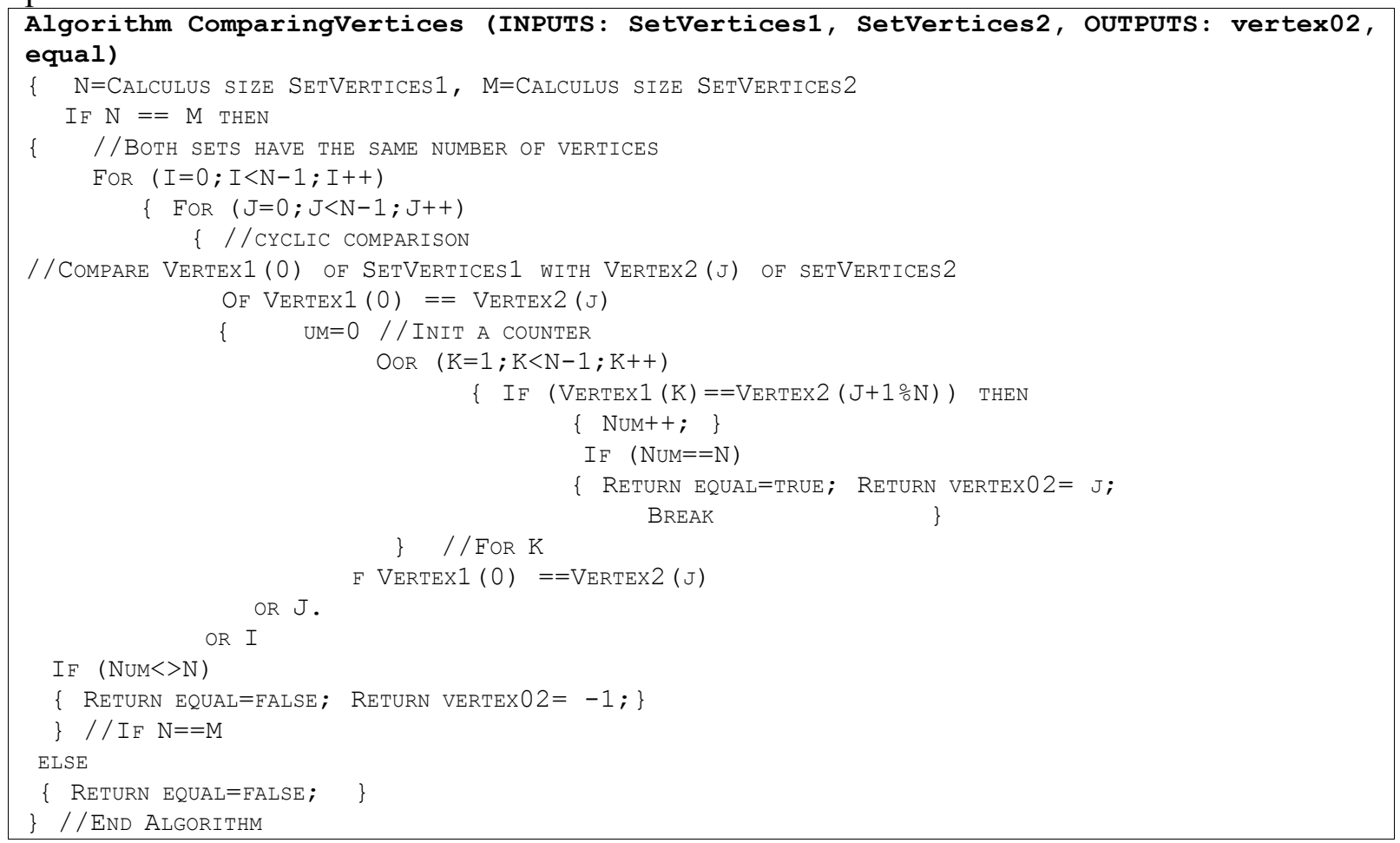


Algorithm 1. Cyclical ordering matching algorithm.

The matching process was begun in this way because the objects with holes that are rotated with respect to the reference object will be described with the holes in a position different from the one given by the reference object although both are the same object. This occurs when an absolute and fixed position is considered for Frank's Cardinal Reference System (CRF) as Figure 3 shows. This happens because it is not known yet how the CRF must be oriented with respect to the parent object. Thus, first of all the program must determine how to orientate the CRF with respect to the parent object. Once it is determined, the description of the second object is completed, and the holes information is compared in order to determine if both objects are finally equal.

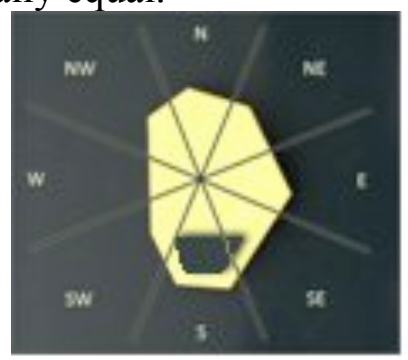

a)

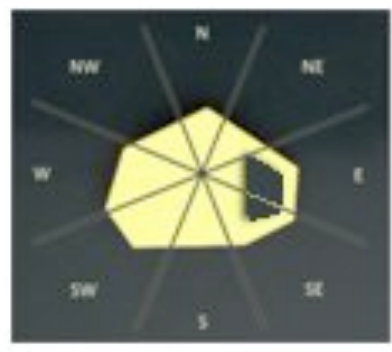

b)

Fig. 3 Two qualitatively equal shapes, but with different orientations. Using an absolute and fixed CRF the holes will have different positions and therefore both qualitative descriptions will be not equal

With respect to the computational cost if $\mathrm{N}$ is the number of vertices of the parent object, $\mathrm{M}$ is the number of vertices of the holes and $\mathrm{K}$ is the number of holes, then for the worst-case need for the construction of a qualitative description of the figures is of the order $\mathrm{O}\left(\mathrm{N}+\mathrm{M}^{*} \mathrm{~K}\right)$, and for the worst-case need for the matching algorithm is of the order $\mathrm{O}\left(\mathrm{N}^{2}+\mathrm{K}^{2 *}(\mathrm{~N}-1)+2 * \mathrm{M}\right)$.

\section{The application designed for the extraction of relevant information from the tessera images}

As previously mentioned, for this application it was necessary to develop an image-processing algorithm in order to obtain the relevant points. The developed algorithm may be classified as hybrid because it uses, first, quantitative techniques to extract the image boundary, and the result is then qualitatively processed to obtain just the points of interest. A full description of the algorithm is provided by Museros and Escrig (2004). In short, the vision system uses segmentation techniques for figure contour recognition, such that a jpg image is taken by a digital camera, and the significant points of the figure are extracted from this by using the well-known segmentation method developed by Canny (1986). However, there are still too many points in the result and, therefore, this set of points is processed qualitatively by slope comparison in order to determine the relevant boundary points.

The computational cost of the hybrid method developed in an image of a number of pixels height * width, with $k$ is the number of points forming the boundaries of the figures in the image, and $d$ the number of relevant points in the image, is $\mathrm{O}$ (height $*$ width $+\mathrm{k}+\mathrm{d}$ ). Since, $k$ and $d$ will obviously be much smaller than height * width, the maximum cost is generated by this product, and it is the computational cost of the Canny algorithm itself. Therefore, no relevant computational cost has been added above the Canny method. At the end of the process gives only the relevant points of the figures in the image, together with information of 
the type of segments of each figure. This information will be what is needed to construct the qualitative string describing each object in the image in accordance with the qualitative shape matching theory. Moreover, the boundaries obtained are more exact than the one obtained using only the Canny Algorithm, with noise removed from the image (see Figure 4).

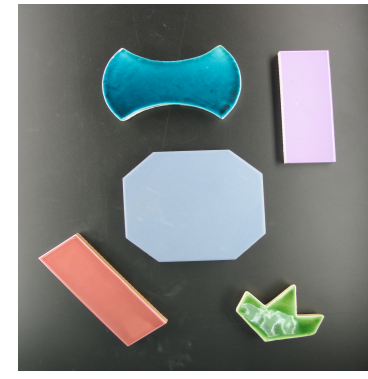

a)

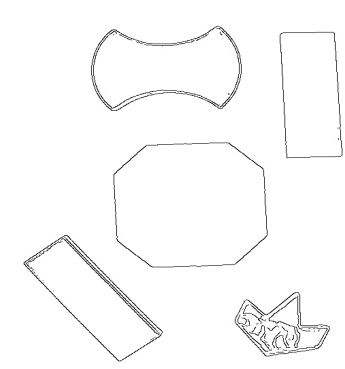

b)

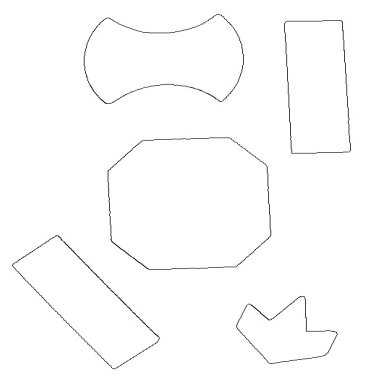

c)

Fig. 4. a) Original image, b) graphical representation of the boundaries obtained using only Canny Algorithm, and c) result of the hybrid method developed applied to the image in a).

\section{Experimental evaluation}

An experiment has been carried out using the map shown in Figure 6a, which is a ceramic mosaic design, created using Photoshop image editing software, and the image with the objects to match shown in Figure 5b, which shows real ceramic pieces, was taken by an industrial camera, model AVT-Guppy F033C.

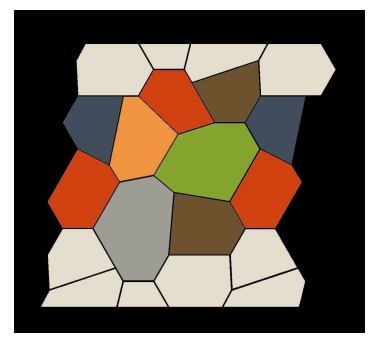

a)

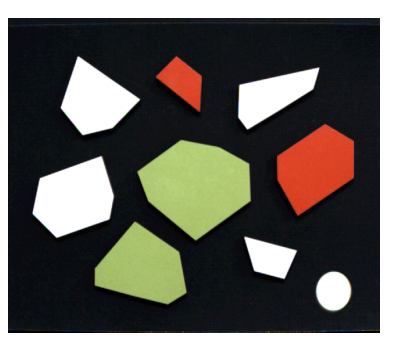

b)

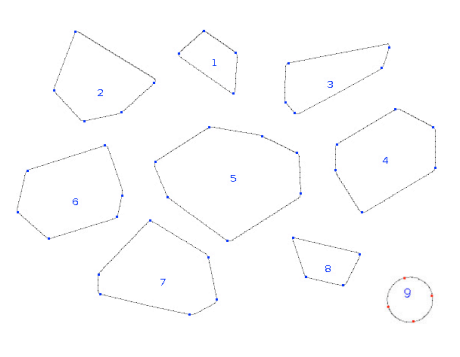

c

Fig. 5 a) Reference image of a mosaic design or map used to carry out this experiment; b) objects to match, and c) relevant points considered to describe the pieces contained in b).

Figure $5 \mathrm{c}$ shows the boundaries and relevant points considered to build the qualitative shape description of the ceramic pieces contained in Figure $5 \mathrm{~b}$. These objects are numbered in order to see in a graphical way which objects have the same qualitative shape as objects in Figure 6a. Figure 6a shows the boundaries and relevant points considered to build the qualitative shape descriptions of objects contained in Figure 5a. In addition, it presents the result of the matching process considering only the shape description by showing a set of numbers inside each object matched. Each number corresponds to the same identification number of the ceramic piece in Figure $5 \mathrm{c}$ that has the same shape. Figure $6 \mathrm{~b}$ shows the same boundaries and relevant points as in Figure 6a, and it also shows the result of the matching process, but in this case, the matching process has been made also considering the color of the objects.

From Figure $6 \mathrm{a}$ and $6 \mathrm{~b}$, it can be concluded that:

1. Without considering the color, objects 2, 3, 5 and 7 in Figure $5 \mathrm{c}$ match correctly with the objects in the ceramic mosaic design with the same shape. Specifically, object 2 has the 
same shape as five objects in the design, object 3 has the same shape as three objects in the design, and objects 5 and 7 have one correspondence (object with the same shape) in the design. Considering the color (Figure 6b), objects 2, 3, 4, 5, 6 and 8 are correctly matched to those objects with the same shape and color in the design. In this case, object 2 is found only twice in the map, because there are only two objects with the same shape and color, the other three objects found in the previous experiment (Figure 6a) differ in color with respect to object 2 .

2. Objects 1 and 8 in Figure 5c have the same shape but different color. Therefore in the experiment that does not consider the color, both should appear in the result, however, the only two objects with the same shape as them are marked only with the number 1 . This is because when one object in the map is matched as equal to another one then it is marked as matched and it is not considered in future comparisons. The same happens with objects numbered 4 and 6 in Figure 5c.

3. In the matching considering the color, objects 7 and 8 are not found in the map due to the color distinction (see Figure $5 \mathrm{a}$ and $5 \mathrm{~b}$ for color differences).

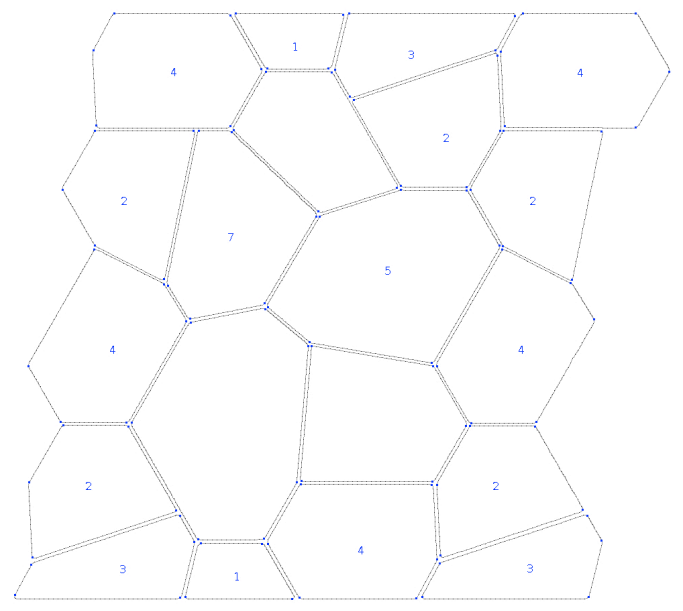

a

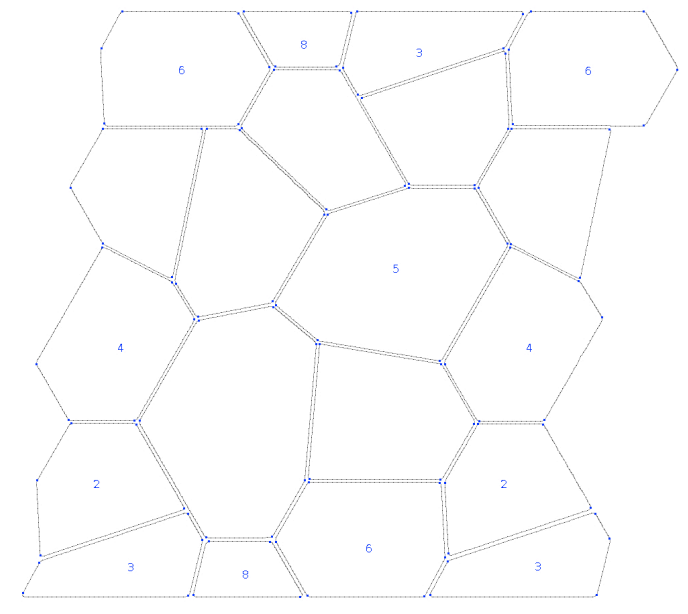

$\mathrm{b}$

Fig. 6 Boundaries and relevant points of the objects contained in the ceramic mosaic design. The numbered objects are the ones with the same shape as those objects with the same identifier in Fig. $5 \mathrm{c}$ in a), and with the same shape and color in b).

Therefore, it is demonstrated that this approach matches objects with the same shape, or with the same shape and color and this is done independently of the rotation and position of the objects in the image. Moreover, our approach can differentiate between objects with the same shape and different color.

\section{The prototype}

The application has been implemented in a system as Figure 7 a shows ${ }^{1}$. The robot arm used is an IRB 140 ABB robot, which is a small 6-axis robot arm, with a payload of up to $5 \mathrm{~kg}$. The

\footnotetext{
${ }^{1}$ A video can be found in: http://www.c4r2.uji.es/index.php?option=com_content\&task=view\&id=63\&Itemid=0
} 
vision system is made up of two AVT-Guppy F033C cameras. All the components are connected using TCP/IP protocol over Ethernet.

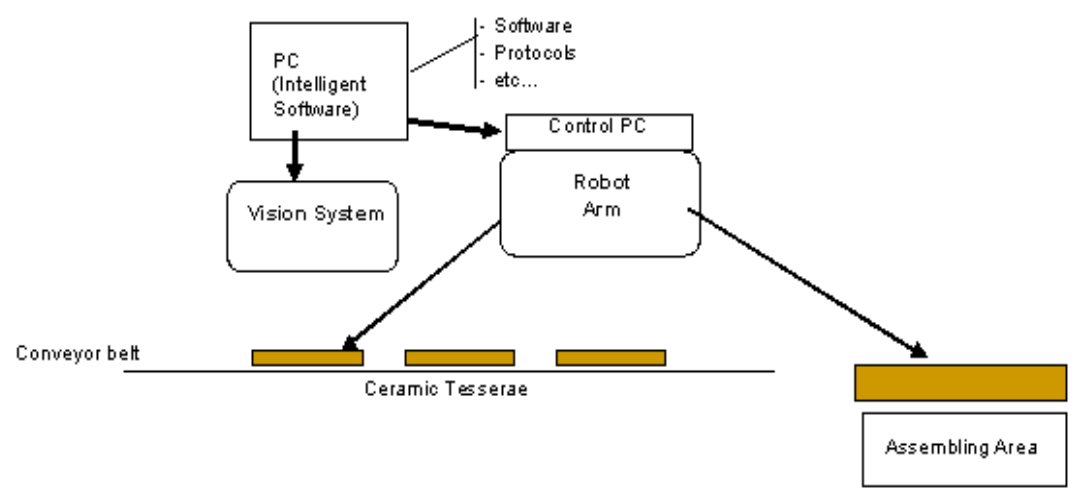

a)

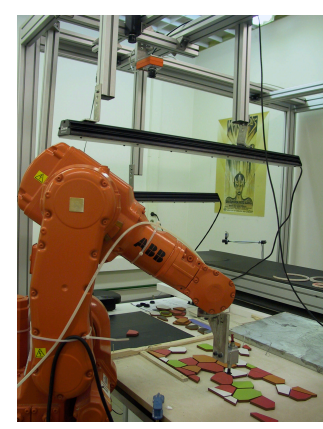

b)

Fig. 7 a) Prototype Diagram; b) Snapshot of the system in our laboratory

The general layout of the system is as follows: The tiles are placed into an input conveyor belt. These tiles will have to be arranged in an output belt so as to correctly fill a mesh by following a predefined design. The process of handling the tiles from the input belt to the output belt will be performed by the robot arm. The two cameras are installed with one on each of the belts. The tool mounted at the end-effector of the robot arm is a vacuum pad, which is connected to a pneumatic vacuum circuit. The vacuum pad diameter is $23 \mathrm{~mm}$. Suction cups provide a reliable technique for moving tiles, because these simple robot tools can handle flat objects weighing from a few grams to several hundred kilograms.

On a PC with Windows XP as the operating system, all the intelligent components explained have been implemented. The intelligent software is developed as a $\mathrm{C}++$ application which contains several $\mathrm{C}++$ classes. There are two specific classes to control the vision system and the robot arm, which implements the drivers of both systems. Then, there are several $\mathrm{C}++$ classes that implement the hybrid method to extract relevant points from an image. Furthermore, there are another set of classes that implement the qualitative shape matching theory, which includes two procedures: i) A procedure that receives the result of the algorithm to extract relevant points from the image and creates the qualitative description of the physical tesserae in the image; and ii) A procedure that implements the matching algorithm.

The final system has been implemented and its general operation is as follows:

1. First a ceramic mosaic design is opened and the qualitative shape description of all the pieces in the mosaic is made.

2. Secondly the camera takes a picture of the physical pieces on the conveyer belt. The driver to communicate with the camera is started.

3. Then the extracting relevant points procedure, which implements the hybrid method, starts and returns the relevant points together with the information on the type of segment.

4. Next, this result is used to qualitatively describe the shapes in the image.

5. The matching algorithm starts and returns which tessera in the image correspond to which piece in the mosaic together with the centroid of the tile and the rotational angle for placing it correctly in the final mosaic. The centroid of the tile is calculated so that the robot arm can pick up the tile using the suction cup at its centroid.

6. Then the communication with the robot arm starts, and the sequence of steps to place each tessera recognized into the final mosaic in the assembly area is sent to the robot. 
7. When this process finishes a new picture is taken, and the process starts again from step 2 . When there are no more physical tesserae to place or the design is finished, the process ends.

The robot control to run the object manipulation processes has been developed by using flexible pick-and-place trajectory sequences. The pick-and-place positions are transmitted from the PC to the robot control unit. The pick position is the centroid of the tile to pick up.

The cameras have been calibrated by using the Tsai camera calibration model (Tsai 1987).

On the output belt, the system assembles a mesh by placing the tiles that correspond to a predefined design with suitable placement and orientation. This mesh is viewed by the system as a set of holes (borders) to fill. Each hole is also described qualitatively using the qualitative shape representation theory presented in this paper. The selection of the mounting point is given by the matching algorithm.

This process has been implemented using real ceramic mosaic designs and tesserae. But the conveyor belt is still stopped. Therefore the real tesserae are placed statically on the conveyor belt surface. The tests done have been carried out with the following parameters: I) The robot arm speed is set to $20 \mathrm{~m} / \mathrm{min}$; and ii) The distance between the input belt and the output belt is 1 meter.

Taking into account these parameters the system needs 3 seconds to pick and place a tile in its corresponding place and orientation in the final mesh, and 2 seconds more to return to the input belt. Therefore it needs 5 seconds for placing each tile, and for instance it will require 8.3 minutes to assemble a mosaic of 100 pieces, which represents a big improvement on the time taken by the hand assembly method currently used

\section{Economic justification of the proposed approach}

If a mosaic factory wants to use this system in an assembly line, it must invest in a PC, a 6axis standard robot arm, two industrial cameras, a vacuum pad, a pneumatic vacuum circuit, and at least one conveyor belt and mesh mounting table. The infrastructure to be bought for the project could also be used in other applications such as painting tiles. The cost of the investment is low in comparison to the benefits that a company may receive from this system. These may include the capability to react quickly to changing markets, with innovative products, a good on-time delivery service and a competitive cost in order to offer good prices, which determines the competitiveness of the manufacturing industry, such as the ceramic, glass and natural industrial sectors, mainly made up of SMEs. In fact, products with specific features and high quality, such as mosaics, often have the best market opportunities. Mosaics represent a high-added value in wall and floor ceramics, glass and natural stone covering products. But due to the fact that nowadays they are hand assembled by crafts people, their manufacturing costs and time to market are higher than other products, and therefore their competitiveness in the market its lower.

Therefore, the economic benefits for the individual SMEs in the ceramic sector are: i) Cost reduction of more than $35 \%$, leading to greater competitiveness; ii) An increase in the product offer by commercializing new and highly value-added products with competitive prices: mosaics, which will enable them to increase sales; and iii) Greater flexibility in order to respond rapidly to market demands and customer requirements.

\section{Conclusions}

The Qualitative Theory of Shape defined in this paper creates object descriptions that remain 
invariant under scaling, translation, and rotation transformations, and it describes shapes qualitatively, which allows us to manage the uncertainty associated with the process of manufacturing objects where two objects are never exactly the same. For instance, in the tile manufacturing process, two tiles can easily have slight differences that can be difficult for the human eye to perceive. As our description is qualitative, two objects only have different shape descriptions if their shapes appear significantly different to the human eye.

The proposed theory has been applied in a laboratory prototype for the automatic and intelligent assembling of mosaic borders in the ceramic industry and it presents several advantages over the use of a quantitative theory, such as the managing of uncertainty.

The current system does not yet consider a moving conveyor belt, so the next step will be to put the belt in motion and integrate that movement into the final system. Once this integration has been performed, operating trials will need to be run of the final system in an industrial environment, integrating this prototype with the other industrial equipment.

Finally, this approach is also being extended in order to enable it to assemble trencadis tile mosaics, which are mosaics made from broken tiles. This extension would be done following a multi-agent approach as in work done by Nejad et al. (2010) and Al-Mutawah et al. (2009). Also, for this purpose, an initial theory for juxtaposing shapes can be found in Museros et al. (2010).

\section{Acknowledgements}

This work has been funded by Fundació Caixa Castelló-Bancaixa under project number PI.IA2008-14.

\section{References}

Al-Mutawah, K., Lee, V.,Cheung, Y. (2009) A new multi-agent system framework for tacit knowledge management in manufacturing supply chains. In Journal of Intelligent Manufacturing 20:593-610.

Belongie, S., Malik, J., Puzicha, J. (2001) Matching shapes. In: 8th IEEE International Conference on Computer Vision (ICCV2001), Vol. I, pp. 454-461, Vancouver, Canada, July.

Bennet, B. (1994) Spatial reasoning with propositional logics. Principles of Knowledge Representation and Reasoning: Proceedings of the 4th International Conference (KR94), San Francisco, CA., Morgan Kaufmann.

Biederman, I. (1987) Recognition-by-Components: A theory of Human Image Understanding. Psychol. Rev., 94, 2: $115-147$.

Canny, J.F. (1986) A computational approach to edge detection. IEEE Trans. pattern Anal. Machin. Intel. 8, 679714.

Chase, S. C. (1997) Modeling spatial reasoning systems with shape algebras and formal logic. In AI EDAM: Artificial Intelligence for Engineering Design, Analysis and Manufacturing, No. 11:4 273-285.

Clementini, E. and Di Felice, P. (1997) A global framework for qualitative shape description. GeoInformatica $1(1): 1-17$.

Cohn, A.G. (1995) A Hierarchical Representation of Qualitative Shape based on Connection and Convexity. Proceedings COSIT'95, Springer-Verlag, 311-326.

Dierks, L. (2004) Making Mosaics: Designs, Techniques \& Projects, A Sterling/Lark Book.

Escrig, M.T. and Toledo, F. (1998) Qualitative Spatial Reasoning: theory and practice. Application to Robot Navigation. IOS Press, Frontiers in Artificial Intelligence and Applications, ISBN 9051994125.

Frank, A. (1992) Qualitative spatial reasoning with cardinal directions. Journal of Visual Languages and Computing, 3, pp. 343-371.

Freksa, C. and Zimmermann, K. (1992) On the utilization of spatial structures for cognitively plausible and efficient reasoning. In: IEEE International Conference on Systems, Man and Cybernetics, Chicago. 
Galton, A. and Meathrel, R. C. (1999) Qualitative outline theory, In IJCAI-99, pages 1061-1066, Stockholm, Sweden.

Galton, A. and Meathrel, R. C. (2000) Qualitative representation of planar outlines, In Proc. Of 14th ECAI, pp. 224-228. IOS Press.

Goldstone, R. L. (1999) Similarity. In: R. A. Wilson and F. C. Keil (Eds.), The MIT Encyclopedia of the Cognitive Sciences. The MIT Press, Cambridge, Massachusetts, USA.

Gottfried, B. (2005) Global feature Schemes for Qualitative Shape Descriptions. In: H.W. Guesguen, Gh. Freksa, G. Ligozat (Ed.), IJCAI-05 WS on spatial an temporal reasoning. Edinburgh, Scotland.

Giannarou, S., and Stathaki, T. (2007) Shape Signature Matching for Object Identification Invariant to Image Transformations and Occlusion. In: W.G. Kropatsch, M. Kampel, and A. Hanbury (Eds.): CAIP 2007, LNCS 4673, pp. 710-717, Springer-Verlag Berlin Heidelberg.

Hoffman, D. D. and Richards, W. A. (1982) Representing smooth plane curves for recognition: implications for figure-ground reversal. In: Proceedings of AAAI-82, American Association for Artificial Intelligence, pp. 5-8.

Hoffman, D.D. and Richards, W.A. (1985) Parts of Recognition. In: Visual Cognition, S. Pinker Editor, MIT Press, pp: 65-96.

Jungert, E. (1993) Symbolic Spatial Reasoning on Object Shapes for Qualitative Matching. Spatial Information Theory. A Theoretical Basis for GIS. European, COSIT'93, LNCS, vol. 716, Springer, Berlin.

Leyton, M. (1988) A process-grammar for shape. Artificial Intelligence, 34:213-247.

Leyton, M. (2005) Shape as Memory Storage. Y. Cai (Ed.): Ambient Intelligence for Scientific Discovery, LNAI 3345, pp. 81-103, 2005. Springer-Verlag Berlin Heidelberg.

Lu, G.J., Sajjanhar, A. (1999) Region-based shape representation and similarity measure suitable for contentbased image retrieval. In: Multimedia Syst. No. 7 (2) 165-174.

Marr, D. and Nishihara, H. K. (1978) Representation and recognition of the spatial organization of threedimensional shapes. In: Proceedings of the Royal Society of London, Series B 200, pp. 269-294.

Mehtre, B. M., Kankanhalli, M.S., Lee, W.F (1997) Shape Measures for Content Based Image Retrieval: A comparison. In: Information Processing and Management, Vol. 33, No. 3, pp. 319-337.

Morse, B. S. (1994). Computation of object cores from grey-level images. In: Ph.D. Thesis, University of North Carolina at Chapel Hill, 1994

Museros, L. and Escrig M.T. (2004). A Qualitative Theory for Shape Representation and Matching Proceedings of the 18 International Conference on Qualitative Reasoning (QR'04), pp. 3-10.

Museros, L., Gonzalez-Abril, L., Velasco, F. and Falomir Z. (2010) A pragmatic qualitative approach for juxtaposing shapes. Journal in Universal Computer Science Vol. 16, No. 11, pp. 1410-1424.

Nejad, H.T.N., Sugimura, N., Iwamura, K., and Tanimizu, Y. (2010) Multi-agent architecture for dynamic incremental process planning in the flexible manufacturing system. In Journal of Intelligent Manufacturing 21: pp. 487-499.

Rosin, P.L. (1993) Multiscale representation and matching of curves using codons. CVGIP: Graphical Models and Image Processing, vol. 55, pp. 286-310.

Rucklidge,W. J. (1997) Efficient locating objects using Hausdorff distance. Int. J. Comput. Vision, No. 24 (3) (1997) 251-270.

Steger, C. (1996) On the Calculation of Arbitrary Moments of Polygons. Technical Report FGBV-96-05, Technische Universität München.

Tsai, R. Y. (1987) Using Off-the-Shelf TV Cameras and Lenses, IEEE Journal of Robotics and Automation, Vol. 3, No. 4, pp. 323-344.

Zhang, D.S., Lu, G. (2002) A comparative study of Fourier descriptors for shape representation and retrieval. In: 5th Asian Conference on Computer Vision (ACCV02), pp. 646-651. 